\title{
Practical use of computational fluid dynamics (CFD) in improving the efficiency of domestic ventilation waste heat recovery systems
}

\author{
Adam Kyte \\ Received: 10 October 2013/Accepted: 15 January 2014/Published online: 4 April 2014 \\ (C) The Author(s) 2014. This article is published with open access at Springerlink.com
}

\begin{abstract}
Efficiency of domestic ventilation waste heat recovery systems (WHRS) depends not only on the amount of waste heat recovered, but also on the energy involved in running fans to drive air through the system. Computational fluid dynamics (CFD) can be a powerful tool for analysing WHRS losses (thus predicting fan energy usage), but the computational effort involved can limit the value of CFD as a practical design tool. This study presents a range of assumptions and simplifications that can be applied to reduce the computational effort associated with the CFD analysis of a WHRS. The importance of experimental validation to assess the effect of errors introduced by the simplifying assumptions is discussed. In an example case, application of the methods presented have allowed total pressure losses (excluding the fixed losses through the heat exchanger) to be reduced by over $50 \%$ in comparison with an initial prototype design, with proportional reduction in fan energy usage. This highlights the value of sufficiently simplified CFD analyses within a typical WHRS product development cycle.
\end{abstract}

Keywords CFD - Computational fluid dynamics - Waste heat recovery $\cdot$ Efficiency

\section{Abbreviations \\ CFD Computational fluid dynamics \\ CPU Central processing unit \\ DNS Direct numerical simulation}

5th International Congress on Energy and Environmental Engineering and Management (CIIEM).

\footnotetext{
A. Kyte $(\square)$

Plymouth University, Drake Circus, Plymouth, Devon PL4 8AA, UK

e-mail: adam.kyte@plymouth.ac.uk
}

\author{
GGI General grid interface \\ RAM Random access memory \\ RANS Reynolds averaged Navier-Stokes \\ RSM Reynolds stress models \\ SST Shear stress transport \\ WHRS Waste heat recovery system(s)
}

\section{Introduction}

Heating (or cooling) and ventilating a building simultaneously can result in significant waste of energy; recent years have seen much development of waste heat recovery systems (WHRS) to transfer heat from foul exhaust ventilation air to fresh air entering a building. In designing a WHRS, an engineer must consider the overall efficiency of the system, offsetting energy savings due to heat recovery against energy used by fans and other components [1]. Performance predictions are commonly made using simulation software (bespoke or generic) based on mathematical functions (empirical or analytical) to model heat loads, heat transfer and many other effects; [2] and [3] present typical examples.

One area that may evade analysis using such techniques is the prediction of the power required to move air through the WHRS; this is highly dependent on the exact geometry of the components within the WHRS and all interconnecting ductwork. Finite volume computational fluid dynamics (CFD) codes can assist with these predictions, but this approach is computationally expensive. Operator and central processing unit hours needed for accurate, rigorous CFD simulations can limit the practicality of this approach as a design tool. It should be noted that a combination of empirical/analytical modelling and CFD may be sensible [4], although the computational effort 
Fig. 1 Schematic diagram of a typical WHRS

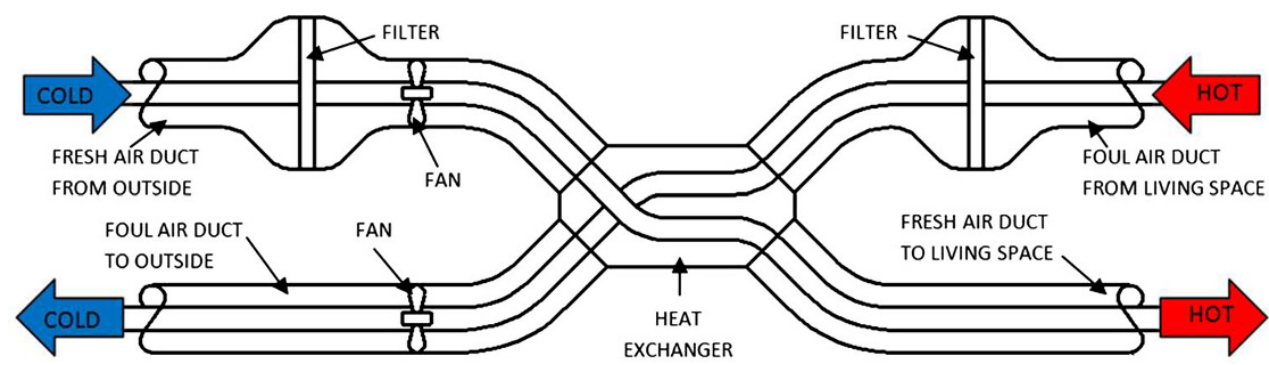

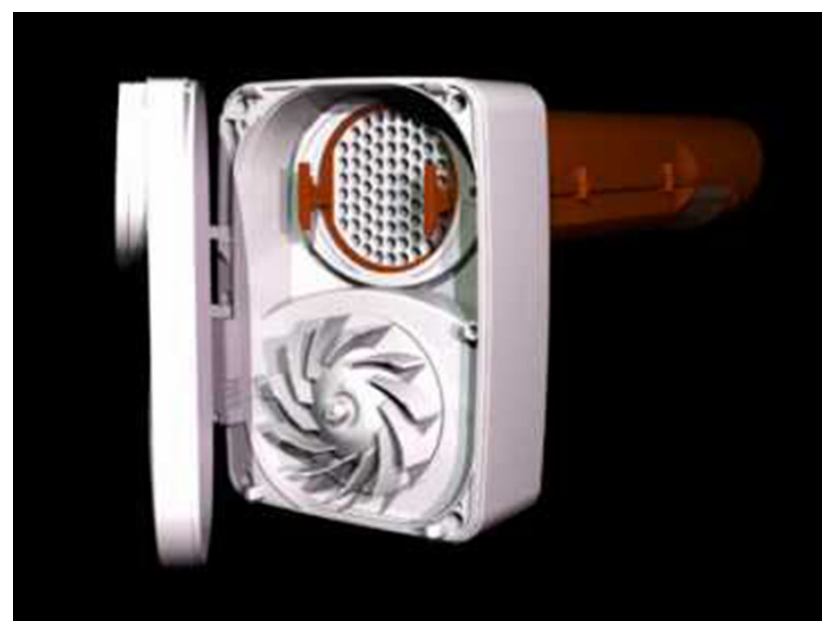

Fig. 2 A compact domestic WHRS featuring a built-in shell and tube heat exchanger

associated with CFD remains an undesirable obstacle. This paper describes a range of simplifying assumptions/ approximations that may be used to speed up CFD simulations to the point that they may be routinely used in WHRS product development cycles. This method has been used to develop one such WHRS using the commercially available CFD code ANSYS-CFX. Details of the actual WHRS design in question are commercially sensitive and, as such, numerical data, device geometry and physical system layout are not presented. However, CFD methods and assumptions are transferable and are discussed in relation to the design of generic systems.

A typical WHRS may include fan(s), filter(s) and heat exchanger(s) as shown schematically in Fig. 1. Often, however, a WHRS may be designed such that these components are close coupled forming a single marketable product (such as that shown in Fig. 2) rather than a disparate collection of components as implied by Fig. 1. Such coupling of components can result in highly irregular air flow paths that defy calculation of pipe losses using traditional empirical methods, leaving the designer little option for performance prediction other than experimentation with physical prototypes or use of CFD software.

Waste heat recovery systems layout and design can vary considerably. The heat exchanger could be of any design, such as a shell and tube or plate type. Fans and filters could be mounted in fresh and/or foul air systems. Fans could be axial, radial or mixed flow. It is clear that minimising electrical power consumption of a WHRS improves net energy saving and should be a design objective. In addition, there are many other (often conflicting) design requirements/constraints, such as minimising cost, noise and space envelope of the system (particularly for retrofit in existing buildings), maximising thermal performance and consideration of maintenance (e.g. filter accessibility and service intervals).

The method by which CFD has been used in a WHRS product development cycle will now be presented, paying particular attention to the CFD simplifications and assumptions necessary to reduce computational effort to a level that is practical in a design context.

\section{Method}

The method presented here is that used for the analysis and development of one specific WHRS and provides a suggested framework for the analysis of other similar systems.

Analysis and design methodology

The general approach was to conduct a baseline CFD analysis (using a range of simplifications/assumptions) and validate this using a physical experiment before using CFD alone for iterative design improvement. The individual steps taken to complete this process were as follows:

1. A first iteration physical prototype was built, having been designed by estimating expected losses using experience and relevant engineering theory (but without the use of CFD). Minor loss coefficients were used to estimate the effects of sudden/gradual expansions/ contractions, bends, etc. This analysis was only approximate; space constraints meant that flow passages differed considerably from the regular geometries typically presented in empirical minor loss data, and the close proximity of adjacent components meant that flow in all cases was far from the empirically assumed fully developed profile. 
2. Computational fluid dynamics simulations of this prototype were conducted using all the assumptions/ simplifications listed later in this paper.

3. Static pressures were measured at multiple locations in the prototype device and compared with CFD predictions. Agreement between experiment and CFD provided confidence in CFD results and justified design development using CFD alone.

4. Computational fluid dynamics simulation domains were split into a number of subsections for analysis. Losses were assessed by finding (mass flow averaged) total pressure at entrance and exit to each subsection. Note that fluid power required to drive a fluid flow may be defined as total pressure loss multiplied by volumetric flow rate (which may be further adjusted to account for fan and motor efficiencies). Total pressure loss is therefore proportional to fan power for a given volumetric flow rate and is therefore an appropriate performance parameter to study.

5. Flow visualisation using CFD post-processing tools was used to determine reasons for total pressure losses, and geometry was iteratively revised accordingly.

6. The effect of each geometry revision was assessed by comparing total pressure losses before and after the geometry change.

7. The iterative process of revising geometry and assessing effects using CFD continued until all significant sources of unnecessary total pressure loss had been minimised (within the bounds of all the aforementioned competing design constraints).

8. A physical prototype of the fully revised geometry was constructed and compared with CFD to confirm the predicted performance improvement.

\section{CFD simplifications/assumptions}

A range of CFD assumptions and simplifications that may be applied in the analysis of a WHRS will now be presented.

\section{Isothermal flow}

Heat transfer need not be modelled in many cases. If an existing heat exchanger is used, the manufacturer's thermal performance data can be used. For custom heat exchangers, empirical correlations [5] can be used. Alternatively, a CFD study of the heat exchanger in isolation from the rest of the system can be conducted. Many heat exchangers comprise multiple adjacent flow channels; appropriate use of symmetry boundary conditions can often permit the modelling of one pair of adjacent channels to give adequate thermal performance prediction of the entire heat exchanger. As an example, the WHRS studied in "Analysis and design methodology" featured a thermoplastic plate heat exchanger of the type shown in Fig. 3. Although there are actually thousands of flow channels in this device, thermal performance can be predicted by analysis of a single pair of the triangular section channels.

With thermal performance separated from flow prediction, assumption of isothermal air is reasonable; density and viscosity variation associated with the small changes in air temperature in a real WHRS could be considered negligible. This also permits CFD study of fresh and foul air systems separately, without the requirement for simultaneous coupled modelling of both sides of the heat exchanger.

\section{Simplified fan behaviour}

Explicit CFD simulation of flow through a fan is computationally expensive. For existing fans, manufacturer's performance curves can be used to determine the power and pressure rise for a given rotation speed and flow rate, so the fan itself need not be explicitly modelled. For example, the fresh air side of the system schematically shown in Fig. 1 may be split into two separate CFD domains as shown in Fig. 4. Domain 1 outlet and Domain 2 inlet utilise mathematical expressions for axial, radial and circumferential velocity as a function of radial position. This approach is applicable to axial, radial and mixed flow fans and crucially allows for swirl to be specified. Expressions for velocity distributions can be determined experimentally (using pitot tubes, hot wire anemometers and wool tufts, for example). The sum of the CFD predictions for the pressure drops through Domains 1 and 2 (plus any pressure difference between the building interior and exterior space) therefore dictates the pressure rise that
Fig. 3 A thermoplastic plate heat exchanger (Recair bv)
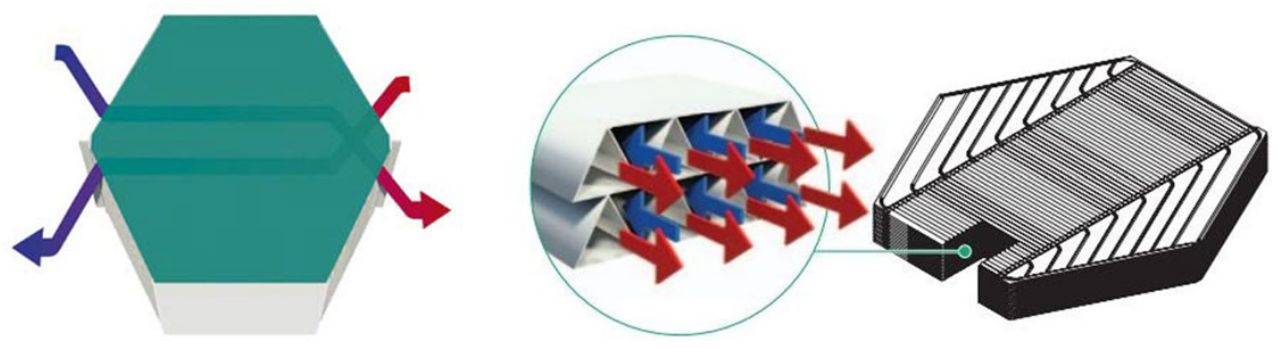


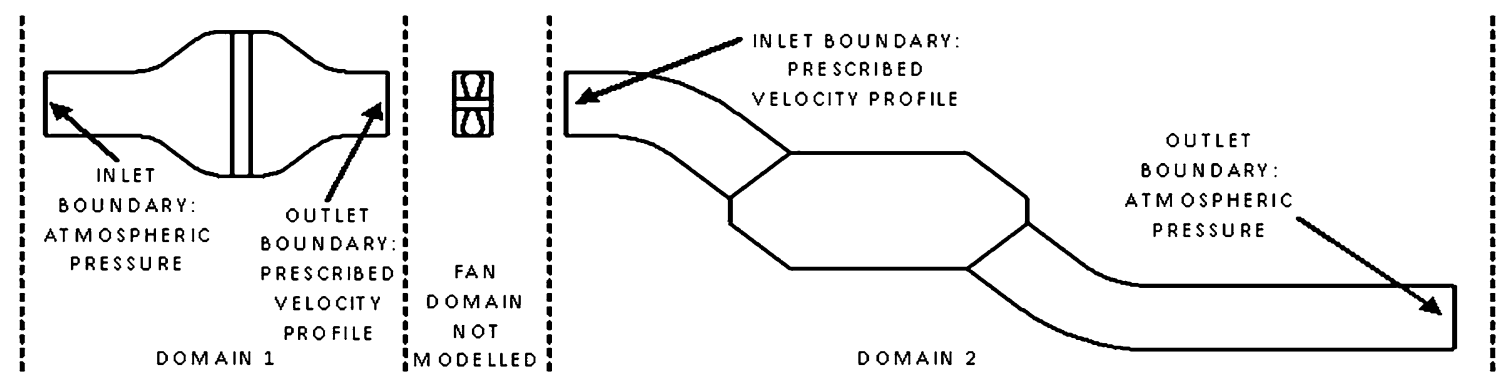

Fig. 4 Fresh air system as two separate CFD domains

the fan must provide. This approach greatly reduces computational effort, but neglects the effects of the geometry adjacent to the fan on the flow within it. If this is a concern, a CFD model of the fan domain alone can be produced, which can later be combined with Domain 1 and 2 models via general grid interfaces (GGIs) [6].

\section{Filters as porous media}

A large filter area is desirable to minimise the mean velocity through the filter which will minimise the pressure drop across it. However, achieving a large filter area may require high expansion and contraction ratios in ductwork. Space constraints may dictate that such expansions/contractions must be quite sudden, thus inducing significant losses. An optimum filter area therefore exists in which combined filter and expansion/contraction losses are minimised. Microscopic flow through three-dimensional filter materials can be complex, but need not be explicitly modelled; ANSYS-CFX permits definition of an isotropic porous loss [7] as follows:

$\Delta P / L=C_{1}(V)+C_{2}\left(V^{2}\right)$

where $\Delta P=$ pressure drop $(\mathrm{Pa})$

$L=$ length in flow direction (filter thickness) (m)

$V=$ velocity $(\mathrm{m} / \mathrm{s})$

$C_{1}=$ linear resistance coefficient $\left(\mathrm{kg} / \mathrm{m}^{3} \mathrm{~s}\right)$

$C_{2}=$ quadratic resistance coefficient $\left(\mathrm{kg} / \mathrm{m}^{4}\right)$

Filter material may therefore be modelled as a single homogeneous porous medium (which does not require fine CFD mesh resolution). Coefficients can be determined experimentally by placing a sample filter of known crosssectional area and thickness in a constant section duct flow. For that given filter material, it is then possible to use CFD to find the optimum cross-sectional area, thickness and orientation to minimise the losses for the combined filter/ duct geometry combination. Filter life can also be considered using this approach; local rate of fouling is related to local flow velocity. By maximising filter area and ensuring as uniform a flow velocity over the whole filter area as possible, filter maintenance intervals will be maximised.

\section{Heat exchangers as directional porous media}

It may not be necessary to explicitly model pressure drop through the heat exchanger. Manufacturer's data, empirical correlations [5] or a simple CFD model of an individual channel can be used. Once the relationship between flow rate and pressure drop is known, it is possible to model the heat exchanger as a porous medium in a similar way to that suggested for the filters. However, this approach does not consider the direction in which flow will enter and exit the heat exchanger. Again, taking the thermoplastic plate heat exchanger shown in Fig. 3 as an example, the baffle orientation in the entrance and exit regions determines the flow entrance/exit angles. ANSYS-CFX permits definition of directional losses [7], whereby losses in a specified streamwise direction are defined as per Eq. 1, and losses in a transverse direction (i.e. perpendicular to the streamwise direction) are defined as the streamwise loss multiplied by a streamwise coefficient multiplier. By setting this coefficient to a high value (e.g. 100), air is effectively forced into flowing in the streamwise direction only.

Correct definition of the streamwise direction is therefore critical. In the example shown in Fig. 5b, the streamwise direction is defined using step functions [8]; $x$ and $y$ components are prescribed to give the required resultant direction in the entrance region where diagonal flow is required, but a step function "switches off" the $x$ component at a $y$ coordinate that represents the point
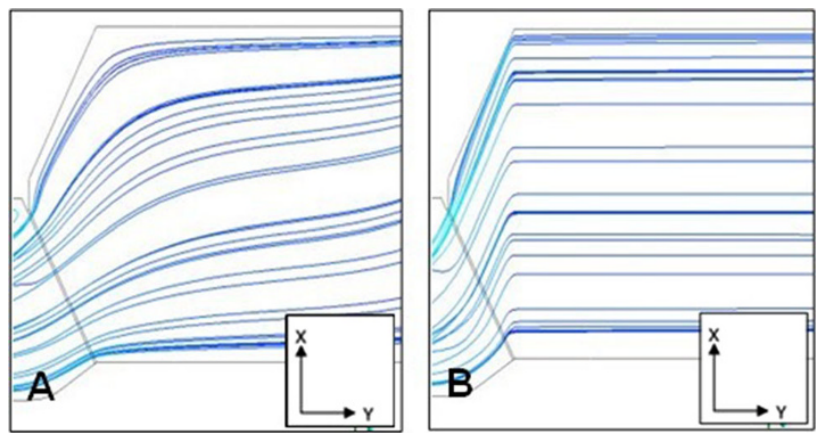

Fig. 5 Flow streamlines through a heat exchanger modelled as isotropic porous media (a) and directional porous media (b) 
where the baffles turn from diagonal to horizontal orientation. Use of the directional loss model therefore correctly defines pressure loss and flow direction within the heat exchanger without the need for explicit modelling of individual heat exchanger channels.

\section{Isotropic turbulence}

In almost all industrial finite volume CFD simulations, direct numerical simulation of all turbulent eddies is computationally impractical, necessitating a Reynolds averaged Navier-Stokes (RANS) approach as detailed in texts such as [12]. One of the most widely used RANSbased turbulence models is the $k-\varepsilon$ model $[9,13]$ which relies on the concept of turbulent viscosity; this in turn makes the assumption that turbulence is isotropic. The $k-\varepsilon$ model is attractive, as it only requires the solution of two equations and a relatively coarse computational mesh. However, the assumption of isotropic turbulence limits the accuracy of results in some cases, notably in curved boundary layers and swirling flows. Reynolds stress models (RSM) [10, 14] still adopt a RANS approach, but solve for each of the six (time-averaged) Reynolds stresses, plus a dissipation term. Reynolds stress models therefore account for anisotropic turbulence but at a cost; the solution of seven (rather than two) equations to model turbulence combined with the requirement for a very much finer computational mesh realistically make for at least an order of magnitude increase in computational demand (and likely very much more).

The poor ability of the $k-\varepsilon$ model to reliably predict boundary layer separation is problematic in a WHRS application; separation results in significant energy dissipation which in turn has a major effect on fan power required to overcome the losses in the system. Reasonable separation predictions are particularly important in the adverse pressure gradient regions associated with diverging flow passages that are almost inevitable in a WHRS. An alternative to $k-\varepsilon$ and RSM is the shear stress transport (SST) turbulence model [11, 15] (effectively a blend between the $k-\varepsilon$ and $k-\omega$ models). Shear stress transport still assumes isotropic turbulence, but has been proven to be much more reliable than $k-\varepsilon$ in prediction of separation. As an example, Fig. 6 shows the different predictions of flow separation past a small obstruction (located in a diverging duct) in one particular WHRS simulation. Note that $k-\varepsilon$ predicts the separation point to be much later than that predicted by SST and thus underestimates the associated losses. This improved prediction is not without cost; the wall function employed by the SST model necessitates a mesh element height adjacent to the wall of around $1 / 50$ of that required by the $k-\varepsilon$ model. However, careful use of mesh inflation layers at walls can

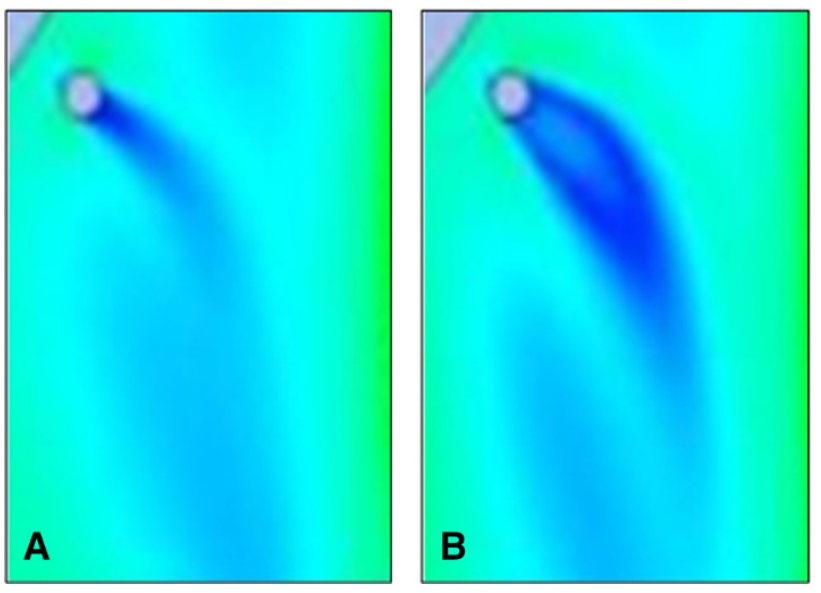

Fig. 6 Flow velocity $($ green $=$ fast, blue $=$ slow $)$ past a small obstruction, $k-\varepsilon$ (a) and SST (b)

ensure that this requirement is met without excessive computational demand.

\section{Steady state}

Transient effects due to flow instabilities (e.g. vortex shedding) may well be present in a WHRS. Designers should normally try to eliminate them as they can be a source of noise, excessive losses and unsteady air flow rates. Transient CFD simulations capable of explicitly modelling such effects are more computationally demanding than equivalent steady-state simulations. They require careful selection of number and length of timesteps to capture the instabilities present, requiring prior estimates of expected frequency. However, steady-state CFD models may be sufficient for design purposes in many WHRS applications; although they cannot explicitly model transient effects, they may still provide a clue as to their presence. Oscillations in convergence graphs of mass, momentum and turbulence residuals that persist despite mesh refinements and steady-state timestep reductions can suggest physical rather than numerical instability. Whilst such observations may not be conclusive, location of such oscillating residuals within the CFD domain can help the designer to identify where geometric modifications could result in steadier (and, most likely, more efficient) flow.

\section{Results and discussion}

The method presented in "Analysis and design methodology" (incorporating the simplifications and assumptions presented in "CFD simplification/ assumptions") was successfully employed in the development of a WHRS. Quantitative results and details of device geometry cannot be presented here for reasons of commercial sensitivity. 
However, qualitative explanation of the results is given to demonstrate the value of the approach outlined in "Method".

Good agreement was seen between initial prototype experimental results and CFD simulations for static pressures at 13 out of 14 measured locations. However, both CFD and experiment suggested significant transient effects at the one location where correlation was poor; experimentally measured pressure showed significant fluctuations whilst CFD showed persistent oscillations in residual error for conservation of mass and momentum. Computational fluid dynamics post-processing revealed a strong vortex structure at that location. It was hypothesised that the instability was due to "wandering" of the vortex core position.

The first geometry revision incorporated redesign of this problematic region to remove the geometric features thought to be instigating the vortex formation. Computational fluid dynamics analysis after this revision showed that the pronounced residual error oscillations were no longer present, suggesting that transient effects may have been reduced. In addition, total pressure loss prediction in this section was some $88 \%$ lower than before the revision: a clear indication of the efficiency improvements that may be possible with an approach that systematically eliminates undesirable flow features.

Several iterations of geometry revision to the entire WHRS layout were conducted. By this point, predictions for total pressure losses for the entire WHRS (excluding the heat exchanger) had reduced by over $50 \%$ in comparison to the initial design case. Note that in this study, the heat exchanger design was a fixed design constraint; total pressure loss across the heat exchanger was therefore unchanged at each iteration and hence excluded from the comparison.

The iterative process was concluded at the point that it was felt that additional possible marginal gains from future iterations would not warrant the associated increase in project cost and duration. The physical prototype of the fully revised geometry was then manufactured and tested.

Experimental results confirmed that predicted reductions in total pressure loss (and thus fan power) were realistic. In addition, system noise and flow unsteadiness had been significantly reduced.

The example given demonstrates the value of CFD analysis in the WHRS design development process. Had the task been performed using experimental testing of physical prototypes alone, then many more prototype iterations would have been required with an associated increase in project cost and duration. In addition, the use of CFD visualisation techniques led to the identification and elimination of undesirable flow features that may not have been found by a trial and error-based physical prototype approach.
The simplifications and assumptions discussed will all have resulted in uncertainty-introducing error into CFD simulations. However, favourable experimental validation has shown that CFD can still be used with sufficient confidence to identify performance trends associated with design revisions. The simplifications/assumptions used ensure that computational expense does not prohibit the use of CFD for typical design cycle durations; the CFD-based development of this particular WHRS took approximately four working weeks to complete using a desktop computer (Intel i5-760 processor, 16 GB RAM).

\section{Conclusions}

Despite inevitable inaccuracies associated with the CFD assumptions/simplifications discussed, CFD can provide useful, timely input into the design of a WHRS, in particular in minimising fan energy usage. Suitable experimental validation is essential, but experimentation alone is unlikely to yield the level of design insight developed using CFD flow analysis and visualisation. In the example case discussed, total pressure losses (excluding those in the heat exchanger) were reduced by over $50 \%$ (between first and last design iterations) for a fixed flow rate. Resulting energy savings clearly show the value of such an approach in today's increasingly energy conscious world.

Acknowledgments Figure 3 provided by Henk Reijneveld, Managing Director, Recair BV, Vijzelweg 165145 NK Waalwijk, Netherlands. http://www.recair.com. All other data and images provided by the author-self funded.

Conflict of interest The example case discussed in this study was conducted as commercial consultancy work for an industrial client. The client has authorised publication of this document. As requested by the client, the document presents the analysis method, but does not give details of commercially sensitive numerical results or geometric details of the particular WHRS in question.

Open Access This article is distributed under the terms of the Creative Commons Attribution License which permits any use, distribution, and reproduction in any medium, provided the original author(s) and the source are credited.

\section{References}

1. Roulet, C.-A., Heidt, F.D., Foradini, F., Pibiri, M.-C.: Real heat recovery with air handling units. Energy Build. 33, 495-502 (2001)

2. Flodberg, K., Blomsterberg, A., Dubois, M.-C.: Low-energy office buildings using existing technology: simulations with low internal heat gains. Int. J. Energy Environ. Eng. 3(1), 1-9 (2012)

3. Chen, Y., Treado, S.: Development of a simulation platform based on dynamic models for HVAC control analysis. Energy Build. (2013). doi:10.1016/j.enbuild.2013.09.016 
4. Zhai, Z., Chen, Q.Y.: Solution characters of iterative coupling between energy simulation and CFD programs. Energy Build. 35, 493-505 (2003)

5. Kays, W.M., London, A.L.: Compact heat exchangers. Krieger, Malabar (1998)

6. SAS IP, Inc: ANSYS 13.0 Help (2010) Modeling guide: 5.3.4 GGI (General Grid Interface) connections, Canonsburg, PA

7. SAS IP, Inc: ANSYS 13.0 Help (2010) Modeling guide: 1.3.2.2.1 isotropic and directional loss models, Canonsburg, PA

8. SAS IP, Inc: ANSYS 13.0 Help (2010) Reference guide: 14.1 CEL mathematical functions, Canonsburg, PA

9. SAS IP, Inc: ANSYS 13.0 Help (2010) Theory guide: 2.2.2.1. The $k$-epsilon model in ANSYS CFX, Canonsburg, PA

10. SAS IP, Inc: ANSYS 13.0 Help (2010) Theory guide: 2.3. Reynolds Stress Turbulence models, Canonsburg, PA
11. SAS IP, Inc: ANSYS 13.0 Help (2010) Theory guide: 2.2.2.6. The Shear Stress Transport (SST), Canonsburg, PA

12. Versteeg, H.K., Malalasekera, W.: An introduction to computational fluid dynamics: the finite method, 2nd edn. Pearson, Harlow (2007)

13. Launder, B.E., Spalding, D.B.: The numerical computation of turbulent flows. Comput. Methods Appl. Mech. Eng. 3, 269-289 (1974)

14. Launder, B.E., Reece, G.J., Rodi, W.: Progress in the development of a Reynolds-stress turbulence closure. J. Fluid Mech. 68(3), 537-566 (1975)

15. Menter, F.R.: Two-equation eddy-viscosity turbulence models for engineering applications. AIAA J. 32, 1598-1605 (1994) 